\title{
An Vision-Based Monitoring System for Accurate Vojta Therapy
}

\author{
Vaishali A. Nimbalkar ${ }^{1}$, Prof. Raskar V.B \\ Student, Department of Electronics and Telecommunication, JSPM's Imperial College of Engineering \& Research, \\ Wagholi, Pune, India ${ }^{1}$ \\ Professor, Department of Electronics and Telecommunication, JSPM's Imperial College of Engineering \& Research, \\ Wagholi, Pune, India ${ }^{2}$
}

\begin{abstract}
One kind of physiotherapy which is known as vojta therapy, is the most significant method to cure the disorder in nervous and musculoskeletal systems. This remedy is proposed by neurologist Mr.V Vojta, hence the name is vojta therapy.He had studied various patients with disorder in nervous and musculoskeletal system,analyzed them and concluded that therapy must be reflective locomotive type which requires selective stimulation to perform on affected body region,so that patient may carry out certain reflexive pattern by its own. The repetition of this stimulation in the long run brings forth the previously blocked connections most of the spinal cord and mind, and after a few sessions, sufferers can carry out those actions without any external stimulation.In this paper we proposed an automatic monitoring system to track infants, it can detect infant ,segment he/her ,extract features and classify them for further treatment.Here we used a RGB-D statistics and to detect infants face a robust template matching based algorithm where as for classification purpose multi-class support vector machine(mSVM) is used.The proposed algorithm is perform on various dataset, which we have obtained from kid health center.Result of this algorithm shows that the proposed method is very efficient and economical for home based therapy.
\end{abstract}

Keywords: Vojta therapy, reflex pattern, support vector machine, robust template maching algorithm.

\section{INTRODUCTION}

In the year fifty Prof Vojta evolved the bases of his diagnostics and therapy, the Vojta principle. The Vojta precept begins out from which is known as "Reflex locomotion". at the same time as seeking out a remedy for infants with cerebral palsy, Prof Vojta found that those kids responded to positive stimuli in certain body positions with habitual motor reactions within the trunk and the extremities. The results of this activation were remarkable: Afterwards, the kids with cerebral palsy ought to first speak more clearly, and after a short time they may get up and stroll more assuredly.Due to the fact the patterns of motion that might be activated in those youngsters with spastic cerebral palsy were now not found in their spontaneous motor activity, however could now be activated incompletely, and ever extra comprehensively and absolutely with numerous, repeated stimulation, Prof Vojta stated that movement improvement within the infantile spastic is probably challenge to functional blockades.

The therapeutic use of reflex locomotion allows basic patterns of motion in sufferers with impaired central nervous structures and loco-motor system to be restored another time.In Vojta remedy, the therapist administers intention-directed pressure to particular zones on the body in a infant who is in a prone, supine or side lying position.there are two motion complexes:Reflex creeping in a prone lying position and reflex rolling from a supine and side lying position. Reflex creeping ends in a type of creeping movement, even as reflex rolling begins from a supine lying position, and transforms, via a facet lying position, into the so-called quadrupedal gait. In reflex locomotion, there may be a coordinated, rhythmic activation of the entire skeletal musculature and a CNS response at diverse circuit ranges. The motor reactions, stimulated by pressure from a described beginning position and which proceed regularly and cyclically, can already be absolutely stimulated and reproduced as frequently as preferred within the new-born

It's been possible to verify that the neurophysiological modifications delivered about through the pathing technique subsisted a certain time after the operating sessions.it is therefore benificial to repeat the remedy severa times regular with day to increase the frequency and the duration of these results .the treatment is recommended 3 to 4 instances /day ; each session lasting 15 to twenty mins ,the best therapeutic condition represents consequently one hour to 90 minutes of every day rehabilization ,divided in parts of about 20 mins.consequently, the therapists also can propose an 


\section{ISO 3297:2007 Certified}

Vol. 5, Issue 6, June 2017

in-home continuation of the remedy. an automated imaginative and prescient based absolute device is needed to research the accurate movements of an babies, in the course of the remedy session. The motive of domestic-based totally absolute remedy evaluation is to provide a proper in-home remedy in place of in-sanatorium treatment. The treatment approach at domestic is not satisfactory beneficial for the fast recovery of a affected person but moreover quite beneficial for those who do no longer have access to a community health facility supplying stated treatment. moreover, the commonplace visits to the therapist's sanatorium add an financial burden as nicely.

In this system, we proposed an automated method for little one's detection and correct recognition of the movement patterns in his/her body additives for the duration of VT, the usage of RGB-D statistics. The technique operates in principal additives, in first it appears for a pre-described toddler's face template extensive picture the use of the sum of square difference (SSD) and cross correlation (CC) based matching algorithms in an efficient way.

2nd, we do the segmentation of the infants frame parts and then extracts various feathers within the segmented body elements to have a study the movements in upper and lower limbs. The observed segmented statistics is used best to become aware of babies lying position during remedy system.In next stage, a multi class support vector machine is used to classify the correct moves of the toddler at some stage in the treatment.

\section{LITERATURE SURVEY}

After studying different IEEE papers, I have amassed some associated papers and documents. some of the factors are mentioned right here:

\footnotetext{
${ }^{1}$ In an editorial authored with the aid of $\mathbf{C}$. Thurau, they developed an method for human detection and simultaneous behavior recognition from pics and picture sequences. An motion illustration is derived via making use of a clustering set of regulations to sequences of Histogram of oriented Gradient (HOG) descriptors of human movement photographs. For novel photo sequences, they had been first locate the human by using matching extracted descriptors with the prototypical motion primitives. Given a chain of assigned movement primitives, that they had built a histogram from observed movement. therefore, behavior have been classified by means of the use of histogram evaluation, deciphering behavior recognition as a hassle of statistical collection evaluation. It enables us for how to come across photo and how we are able to classify it.
}

${ }^{2}$ In the area of picture proccessing, O. Hosseini Jafari, Dennis Mitzel, Bastian Leibe have been studied a actual-time RGB-D primarily based multi-character detection and monitoring system appropriate for mobile robots and head-worn cameras. their goal changed into to combines RGB-D seen odometry estimation, region-of-interest processing, floor aircraft estimation, pedestrian search for tracking components proper into a robust vision tool that runs at greater than $20 \mathrm{fps}$ on a computer. specially, they proposed to apply distinct detectors for particular distance degrees. For the close to variety (as plenty as $5-7 \mathrm{~m}$ ), to present a very speedy depth-based upper-body detector that lets in video-rate system overall performance on a single CPU core while carried out to Kinect sensors. in order to cover moreover farther distance levels, they optionally brought an look-based full-body HOG detector (running at the GPU) that exploits scene geometry to restrict the search space. they worked with each Kinect RGB-D input for indoor settings and with stereo depth input for out of doors conditions.

Chang Y.J.; Chen S.F.; Huang J.D.had amazing research and outcomes on A Kinect-based totally system for physical rehabilitation: A pilot examine for young adults with motor disabilities. They had gift a new approach for hand gesture reputation based on an RGB-D sensor. It takes advantage of depth statistics to cope with the maximum commonplace problems of traditional video-based totally hand segmentation strategies: cluttered backgrounds and occlusions. The set of rules additionally uses colour and semantic facts to because it should be choose out any type of arms present inside the picture. Ten special static hand gestures are recognized, consisting of all one of a kind combos of spread arms. furthermore, movements of an open hand are observed and 6 dynamic gestures are identified. the number one benefit of this technique turned into the freedom of the consumer's hands to be at any feature of the photograph with out the need of sporting any precise clothing or additional devices. besides, the complete approach could be accomplished without any preliminary training or calibration. Experiments executed with extraordinary users and in precise environments display the accuracy and robustness of the approach which, moreover, may be run in real-time.

\section{RELATED WORK}

An proposed method algorithm works in two main steps: first step consist of Image Preproccessing, infant detection, verification where as at second stage we have to perform segmentation of detected infant and feature extraction along with classification of image with the help of multi- class support vector machine. 


\section{International Journal of Innovative Research in} Electrical, Electronics, Instrumentation and Control Engineering

ISO 3297:2007 Certified

Vol. 5, Issue 6, June 2017

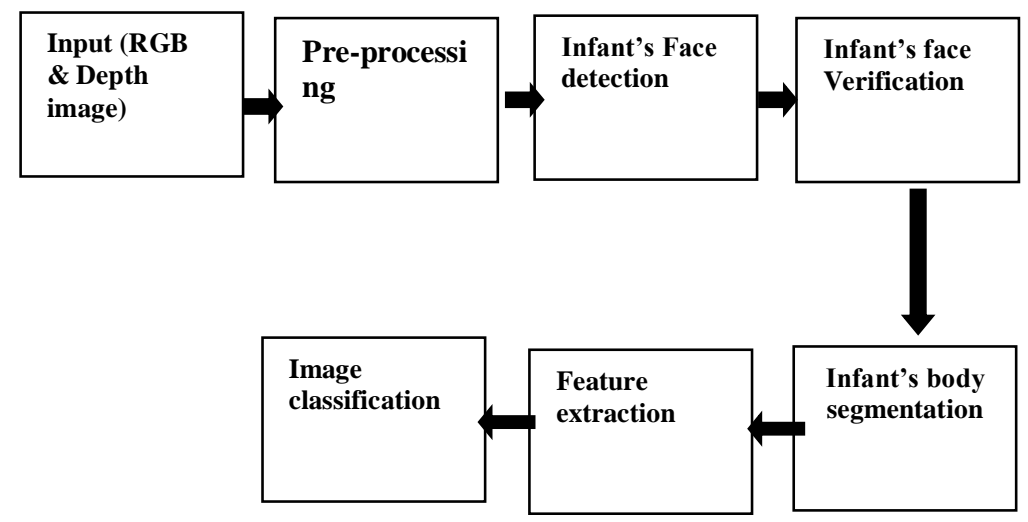

Fig 1. Overview of Proposed Method

\section{A. Input Image}

The first step of the algorithm is to take a live picuters of infants(patients) continuously with the help of efficient camera, which are further helps in treatment. For this purpose here we are going to use kinect camera .

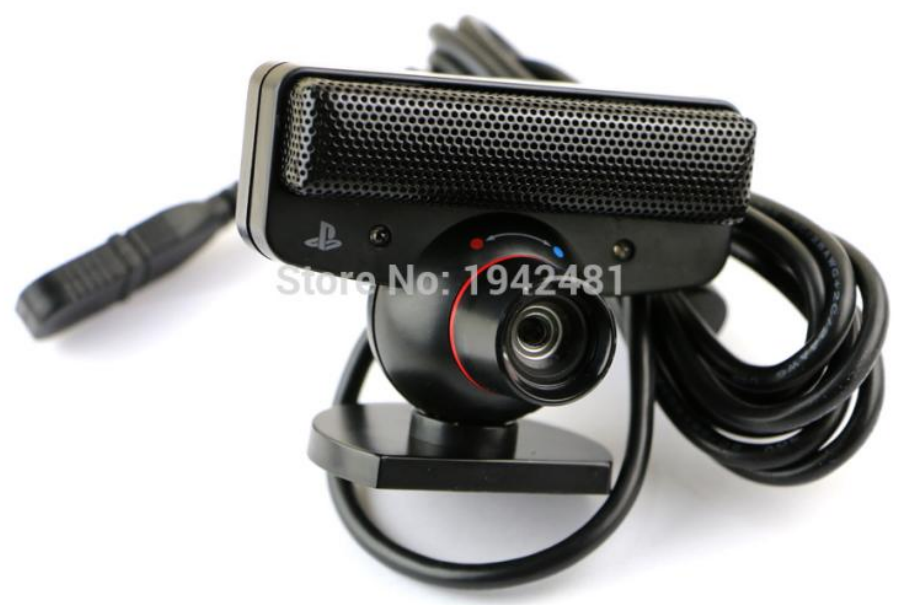

Fig 2. Kinect camera used to access images

A Kinect digital camera is quite moderately priced and can be prolonged far beyond gaming. it is composed of RGB camera, a 3-d depth sensor that offers the space records of object from digital camera, and a pivot which may be tilted each up or down route for sensor adjustment. numerous researchers have implemented the Kinect as assistive generation at ambient assisted living and rehabilitation locations. A device for in-domestic rehabilitation the usage of Dynamic Time Warping (DTW) set of regulations and fuzzy accurate judgment. The evaluation is performed at the trajectory of joints and the time period within the final touch of positive workout. Exell et al. implemented the skeletal tracking data to study the rehabilitation in the top limbs. Chang et al. proposed a system that used the motion tracking of 6 higher limbs for rehabilitation. For validation they used the outputs of OptiTrack as ground truth and in contrast them to the outputs of Kinect. Yao-Jen et al. advanced a Kinect-primarily based definitely rehabilitation machine to help the therapists of their paintings to cope with the kids affected by motor disabilities. They used the motion tracking statistics to investigate the rehabilitation requirements and to permit the therapist to view the rehabilitation progress.

\section{B. Image Pre-Processing}

After collecting the images from kinect camera the next step is to process that image in order to obtain a black and white image so that it may results in ease to detect edge.At first the RGB image is pass thro' RGB to gray scale converter with the help of matlab software, again we have to convert gray scale image to Black and white Image using Grayscale to BW conveter in order to edges in current image 


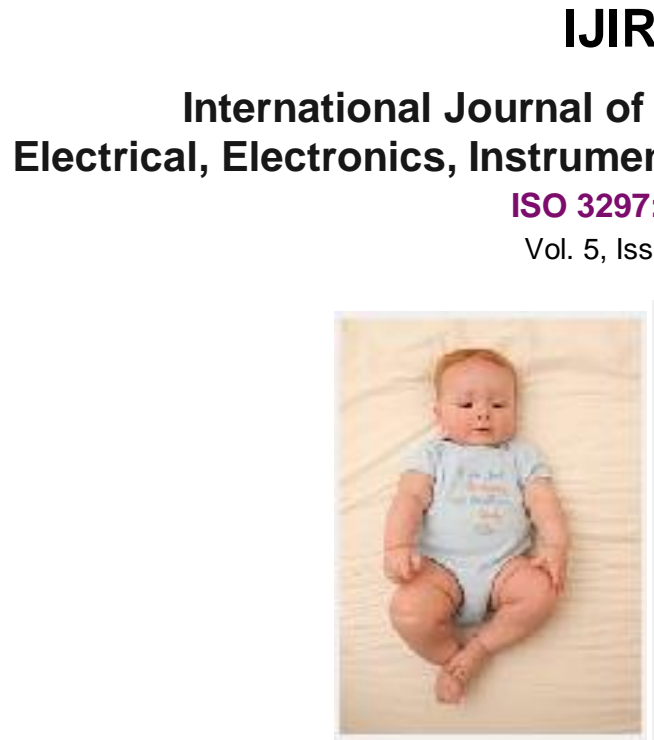

(a)

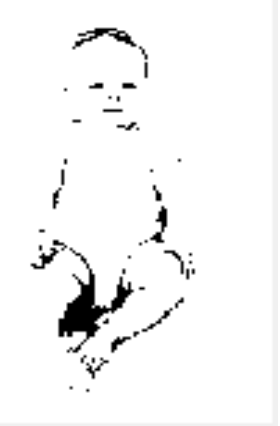

(b)

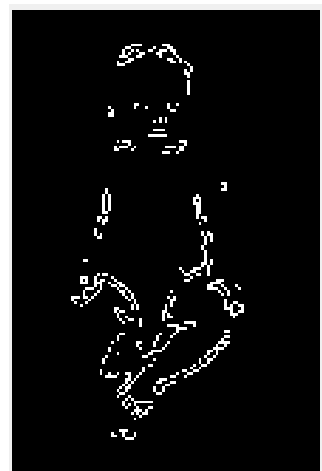

(c)

Fig 3. ouput of preprocessing stage (a) An RGB image from kinect camera ,(b) gray scale image of (a), (c ) Black and white conversion for (b)

C. Infants Face Detection

A massive form of algorithms were proposed for human detection in RGB and depth pictures. numerous RGB based totally absolutely techniques normally used both object features in conjunction with histogram of orientated gradient (HoG) descriptors or motion features like spatio-temporal interest elements (STIPs) for the stated reason. a few different strategies exploited form-based capabilities and the interest points extraction from the scene together with scale-invariant feature transform(SIFT) i.e characteristic transform . despite the fact that maximum of those methods claim excessive accuracy in human detection, visual information still stays a difficult project due to problems because of illumination modifications, occlusions, as nicely as complicated and cluttered background. As an opportunity, the intensity information has severa benefits over the seen information because it offers 3-D structural facts of the scene. moreover, the layered shape of the intensity statistics gives extra information and important cues for human detection and their movement recognition. Due to these advantages, many algorithms for human detection have also been proposed in depth photographs. Zhu et al. proposed a human detection by the usage of tracking his/her face and torso extensive pictures. They proposed the circle and field fitting to come across the face and torso respectively. A support vector machine has been trained to discriminate the human face and shoulders for detection features. The human face frame template is proposed in to slide over the depth photograph and come across the human vicinity using the Euclidean distance. Xia et al. proposed a human-face template for the detection of a human tremendous images the use of the 2-D chamfer matching approach.

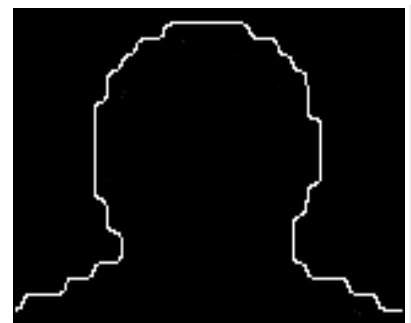

(a)

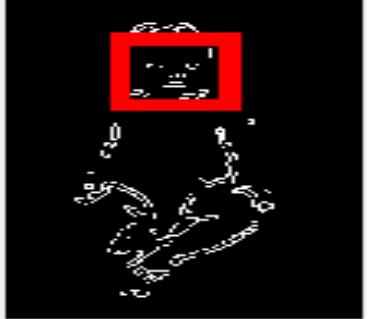

(b)

Fig.Output of face detection stage (a) refference matching template,(b)Detected face with reffernce to (a) 


\section{ISO 3297:2007 Certified}

Vol. 5, Issue 6, June 2017

\section{PROPOSED METHOD}

The proposed approach works in steps. First, the infants are detected significant records the usage of the vicinity of their face characteristic. In a given 2D array of depth information from Microsoft Kinect, we reduced the noise and smoothed the array for later approach. The edges statistics in the intensity records is implemented to discover the candidate positions of the little one's region. The proposed technique scans during the complete image and exploits the relationship among the sum of squared differences (SSD) and cross correlation (CC) based totally matching algorithms, to find the viable areas of the infant's face each of the detected face region is confirmed through a 3D face -model, which applied each the edge and relational depth exchange records from authentic intensity array. After verification, a region developingv set of rules is exploited at the detected region to section the little one's frame region from the captured scene. We finished the calibration of intensity and RGB snap shots the usage of the digital digicam extrinsic and intrinsic parameters to phase the infant's body vicinity from RGB image as well.

In next step, We extract the numerous capabilities in the segmented toddler's body region to capture the actions in higher and lower limbs. The visible segmented data is carried out to handiest perceive the infant's lying role in some unspecified time in the future of the remedy process. in the elegance level, a multi-magnificence support vector system is used to categorise the precise actions of the infant at some point of the treatment.

The matching measure is determined on every pixel of the threshold image which lies beneath the template. For the matching of the little one's head, we implemented the SSD and CC primarily based matching algorithms. let us define the brink image as $\mathrm{E}$ and template photograph $\mathrm{T}$ with size $\mathrm{h} \times \mathrm{w}$. The equation of SSD may be written as:

$$
S(x, y)=\sum_{i=0}^{h} \sum_{j=0}^{w}(E(x+i, y+j)-T(i, j))^{2}
$$

wherein $\mathrm{s}$ is the sum of square differences, evaluated at function $(\mathrm{x}, \mathrm{y})$ of $\quad \mathrm{E} \& \mathrm{i}, \mathrm{j}$ represents $\mathrm{x}, \mathrm{y}$ places . On expanding equation (1) we get,

$$
\begin{aligned}
& S(x, y)=\sum_{i=0}^{h} \sum_{j=0}^{w} E^{2}(x+i, y+j)+\sum_{i=0}^{h} \sum_{j=0}^{w} T^{2}(i, j) \\
& -2 \sum_{i=0}^{h} \sum_{j=0}^{w} E(x+i, y+j) T(i, j)
\end{aligned}
$$

In Eq. (2), the primary term is the sum of squared values in the edge image, the second one time period belongs to the template image and the $1 / 3$ time period is twice the correlation between the photo patch and template. be aware that 2 nd term is contant term .let us count on that third term is approximately contant, so last term is cross correlation:

$$
C(x, y)=\sum_{i=0}^{h} \sum_{j=0}^{w} E(x+i, y+j) T(i, j)
$$

in which $\mathrm{C}$ is cross correlation.it could be effortlessly found from Eq. (2) that the Euclidean distance among the patch of edge picture and template decreases because the correlation (i.e.,similarity) between the photograph patch and template increases.

\section{EXPERIMENTAL RESULTS}

We acquire more than 9000 frames containing the actions of 10 children of various ages (4 week to 1 year) and both genders ( 6 males and 4 females) from children health center.The digital camera turn out to be located on a tripod, at the height of two meters and with an attitude of 45 。 from the desk floor (wherein the little one is lying for remedy), as shown in Fig. The placing grow to be selected to be according with the advice for capturing the high-quality information excellent (i.e., moves patterns in top and lower limbs) with minimal occlusion. For each affected individual the therapy and similarly immoderate sub-bins are used for function extraction. consultation typically lasted among 15-20 minutes, each the RGB and depth frames are recorded at a $640 \times 480$ resolution.

\section{A. Detection and Verification result}

The proposed detection set of policies is applied on our collected challenging dataset for little one's detection using excellent depth data. We done the detection fee of $83.29 \%$ with a small faux alarm of $1.27 \%$. The purpose for the drop in detection fee is because of the occlusion at the region of the infant's face. although it was pre-decided with the therapists to 


\section{IJIREEICE \\ International Journal of Innovative Research in Electrical, Electronics, Instrumentation and Control Engineering \\ ISO 3297:2007 Certified \\ Vol. 5, Issue 6, June 2017}

use their arms with the minimum occlusion of the little one's face shape, the scenario was specially regularly faced while the remedy was given in supine mendacity position

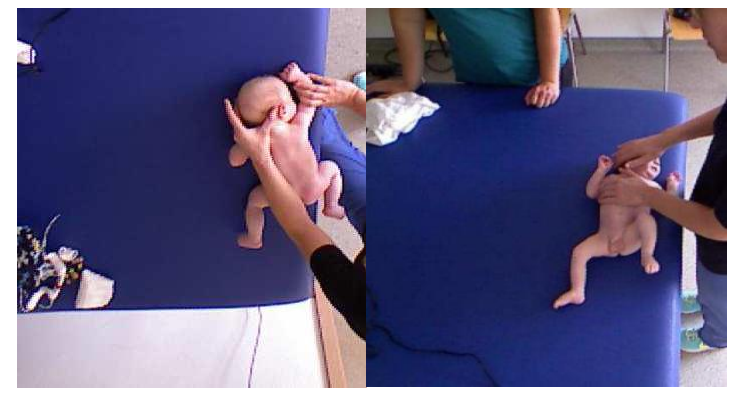

(a)

(b)

Fig. Example of head poses at some stage in the therapy. (a) an infantwith clean head structure in prone lying position, (b) occlusion inside the toddler's head structure may be located in supine lying role because of the therapist's hand.

\section{CONCLUSION}

Here I have given the paper on implimenting few steps of An Vision Based Monitoring System For Accurate Vojta Therapy In this proposed system, the technique of infants detection using kinect camera and canny edge detector with the help of robust template matching algorithm is implemented .The Refference template is slided over pre- processed captured infants image in order to detect infant in picture. Result shows that this technique is reliable ,efficient and econimical way to treat infant at home based remedy .

\section{ACKNOWLEDGMENT}

We want to acknowledge Principal, Head of department and guide of our project for all the support and help rendered. To express profound feeling of appreciation to their regarded guardians for giving the motivation required to the finishing of paper.

\section{REFERENCES}

[1] Muhammad Hassan Khan; Jullien Helsper; Zeyd Boukhers; Marcin Grzegorzek "Automatic recognition of movement patterns in the vojta-therapy using RGB-D data” in 2016 IEEE International Conference on Image Processing (ICIP).

[2] C. Yang et al., "Shape-based classification of Environmental microorganisms,"in ICPR, 2014.

[3] M. N . A. Khan; A. Zuberi, "Fast and accurate localization of human faces", in IEEE International Symposium on Communications and Information Technology, 2004. ISCIT 2004

[4] C. Thurau, "Behavior histograms for action recognition and human detection," in Human Motion-Understanding, Modeling, Capture andAnimation, 2007.

[5]https://nydnrehab.com/what-we-treat/neurological-conditions/cerebralpalsy/2015

[6]https://www.ncbi.nlm.nih.gov/pmc/articles/PMC5332993/2017

[7] C. Shih, M. Chang, and C. Shih, "A limb action detector enablin people with multiple disabilities to control nvironmental stimulation through limb action with a nintendo wii remote controller," in Research in Developmental Disabilities, 2010, pp. 1047-1053.

[8] Chang, Chih-Chung, and C. Lin, "Libsvm: A library for support vector machines," in ACM Transaction on Intelligent Systems and Technology, 2011.

[9] O. Hosseini Jafari, D. Mitzel, and B. Leibe, "Real-time rgb-d based people detection and tracking for mobile robots and head-worn cameras," in IEEE International Conference on Robotics and Automation, 2014.

[10] Y. Zhu and K. Fujimura, "Constrained optimization for human pose estimation from depth sequences," in IEEE Asian Conference on Computer Vision, 2007, pp. 408-418.

\section{BIOGRAPHIES}

Vaishali A. Nimbalkar has Completed B.E in Electronics and Tele-Communication in Imperial college of engineering and research, J.S.P.M. Wagholi, Pune and pursuing ME in ICOER JSPM Wagholi, Pune.

Prof. V. B. Raskar, Professor in ICOER JSPM Wagholi, Pune. 